\title{
Posibilidad Lógico-epistemológica del Modus Tollendo Tollens en la Investigación en las Ciencias de la Educación*
}

\author{
Logical-epistemological possibility Tollendo Modus \\ Tollens in Research in Science Education
}

\section{Possibilidade Lógico-epistemológica do Modus Tollendo Tollens na Investigação nas Ciências da educação}

\author{
Luis Francisco Ochoa** \\ Universidad Santo Tomás, Bogotá, Colombia \\ Asociación Colombiana para el Avance de la Ciencia \\ Astrid Bastidas ${ }^{\star \star *}$ \\ Universidad La Gran Colombia, Bogotá, Colombia \\ Universidad del Tolima, Colombia
}

Resumen. La propuesta de Karl Raimund Popper (1902-1994), para establecer una solución al problema de la inducción en ciencia a través de la falsación (uso del Modus Tollendo Tollens), presenta una serie de

Artículo de reflexión de tema sobre el uso del método Modus Tollendo Tollens.

** Lic. Psicología-Pedagogía, Mg. en Educación, Esp. Filosofía de la Ciencia. Profesional Asesor en formación por proyectos en el Centro de Diseño y Metrología del SENA-Regional Distrito Capital. Miembro Titular y Tutor Científico de la Asociación Colombiana para el Avance de la Ciencia. Docente Director de Trabajo de Grado de la Universidad Santo Tomás, Bogotá, Colombia. E-mail: luisochoa@ustadistancia.edu.co

*** Lic. Ciencias Sociales, Mg. en Educación, Esp. Enseñanza de la Historia, Esp. Filosofía de la Ciencia. Docente Facultad de Derecho Universidad La Gran Colombia y de la Especialización en Pedagogía de la Universidad del Tolima. Miembro Titular de la Asociación Colombiana para el Avance de la Ciencia.E-mail: bastidasastrid@gmail.com 
inconvenientes metodológicos, epistemológicos y algunos históricos, para ser aceptada como posibilidad procedimental, para la investigación en las Ciencias Humanas y Sociales y en especial en las Ciencias de la Educación. Este ensayo presenta los argumentos que sustentan esa tesis.

Palabras Clave. Filosofía de la lógica, Filosofía de la Ciencia, Modus Tollendo Tollens, falsacionismo, Ciencias Humanas y Sociales, Ciencias de la Educación (Tesauro Unesco).

Abstract. The proposed Karl Raimund Popper (1902-1994), to establish a solution to the problem of induction in science through falsifiability (use of Modus Tollens tollendo), presents a number of methodological problems, some epistemological and historical, to be accepted as procedural possibility for research in the humanities and social sciences and especially in the Education Sciences. This paper presents the arguments supporting that thesis.

Keywords. Philosophy of logic, Philosophy of Science, Modus Tollens, falsifiability, refutability, Humanities and Social Sciences, Science Education (Thesaurus Unesco).

Resumo. A proposta de Karl Raimund Popper (1902-1994), para estabelecer uma solução ao problema da indução em ciência através falseabilidade (uso do Modus Tollendo Tollens), apresenta uma série de inconvenientes metodológicos, epistemológicos e alguns históricos, para ser aceita como possibilidade procedimental, para a investigação nas Ciências Humanas e Sociais e em especial nas Ciências da Educação. Este ensaio apresenta os argumentos que sustentam essa tese.

Palavras Chave. Filosofia da lógica, Filosofia da Ciência, Tollendo Tollens, falseabilidade, Ciências Humanas e Sociais, Ciências da Educação (Tesaurus Unesco).

\section{Introducción}

El inductivismo como método de investigación, parte de hechos empíricos irrefutables que poco a poco se constituyen en una estructura axiomática de afirmaciones deducidas, de las observaciones de las cuales se construyen enunciados particulares, que luego pasan a enunciados universales a través de supuestas inferencias lógicas. Estas inferencias 
(que se conjeturan como lógicas) conducen a la elaboración de hipótesis, leyes o teorías. Los enunciados anteriores fueron aceptados por los filósofos empiristas del positivismo del siglo XIX y positivistas lógicos del XX, como el método válido para el desarrollo y avance de las teorías en ciencia. Su punto de partida es la observación para la elaboración de teorías, que para este caso se entienden como:

...un conjunto de enunciados hipotéticos (conjeturales) articulados lógica y semánticamente, que tienen como propósito describir, explicar, predecir, comprender y transformar- operar-instrumentar los fenómenos (variables o factores) de un determinado sector del mundo real, tomados como objeto de investigación dentro de una ciencia o conjunto de ciencias (puras o aplicadas), mediante observaciones sistemáticas, verificaciones, experimentos, contrastaciones empíricas, interpretaciones hermenéuticas, demostraciones racionales o desarrollos tecnológicos (pruebas de hipótesis, o refutaciones experimentales, demostraciones racionalmente sometidas a crítica, diseños y desarrollos) (Ochoa, 2013, p. 4).

Las teorías científicas están montadas en el lenguaje. Es decir, en un sistema de comunicación estructurado que posee un contexto de uso (comunidades de investigadores de las diferentes ciencias) y principios de combinación de orden formal, tanto en expresiones naturales como artificiales. Este sistema de comunicación estructurado tiene como base mínima los enunciados que se entienden como emisiones en una lengua particular con significado (sentido), que puede ser verdadera o falsa, con respecto a los fenómenos (estados de cosas) de un sector del mundo real. La articulación de los enunciados mediante las leyes de la lógica, permite, junto con la unidad de significado, la inteligibilidad de las teorías dentro de las comunidades científicas.

Una teoría científica, usualmente, es una producción del intelecto humano con carácter provisional, cuyo propósito es proporcionar entendimiento adecuado de los fenómenos para solucionar un problema de orden formal ó práctico. Su función es exponer el comportamiento de los fenómenos de manera explicativa por causa eficiente y comprensiva por causa final; por lo tanto, su valoración se refiere, de una parte, a establecer la probabilidad de ocurrencia $y$, por otra, a develar el significado e intencionalidad de tales fenómenos. Lo anterior, en razón a que los enunciados en ciencias físico-naturales (y algunas veces las humanas y sociales) tan solo pueden alcanzar grados continuos de probabilidad bajo límites de superior e 
inferior mediante investigaciones cuantitativas; y logran comprensiones parciales e históricamente ubicadas en las ciencias humanas y sociales mediante investigaciones cualitativas.

El hecho de que una predicción, en ciencias físico-naturales, sea observada de acuerdo a lo predicho en una teoría, no significa que pueda tomarse como prueba contundente, ya que el mismo resultado experimental puede haber ocurrido por motivos que nada tienen que ver con la teoría propuesta. De tal forma, que si se toma el camino de la verificación empírica ${ }^{1}$ como aplicación del principio de inducción, se requiere de una sustentación lógica, es decir, deductiva, para justificar dicha inducción. Esto siempre ha presentado problemas en las ciencias humanas y sociales por su dificultad de poder establecer leyes y teorías generales en tales ciencias.

Con el propósito de resolver el problema de la inducción, en el cual se cuestiona si el resultado de la aplicación de métodos inductivos produce conocimiento válido y verosímil; y si esto permite justificar lógica y metodológicamente las teorías científicas; Popper plantea en Logic der Forschung ${ }^{2}$ de 1934, pero fechada en 1935, el método de la falsación, en el cual, una teoría es una hipótesis tentativa que requiere ser contrastada de forma rigurosa por medio de la observación y la experimentación. En caso de que no supere las pruebas observacionales y experimentales, se elimina y reemplaza por otra; esto hace que la ciencia "progrese» gracias a las conjeturas y refutaciones, a fin de que sólo sobrevivan las teorías más aptas.

Popper considera que el método inductivo genera incoherencias al necesitar presentar un enunciado universal y con ello, reaparece el problema de tener que crear hipótesis auxiliares para lograr acomodar una teoría de acuerdo a los datos y es por ello que para justificarlo, necesita utilizar inferencias inductivas de orden superior aplicadas deductivamente para justificar las de orden inferior, aspecto que deja sin bases a la inducción al intentar un fundamento en la experiencia a través de una regresión infinita. De esta manera, no existe para Popper algo que pueda llamarse inducción.

1 Se refiere a que un enunciado sólo tiene significado cognitivo si su verdad o falsedad puede ser comprobada mediante observaciones empíricas, o que las cosas son como en la proposición se dice que son, por lo tanto prueban su verdad o falsedad.

2 En español Lógica de la Investigación Científica. La cual se citará aquí en su $5^{a}$. reimpresión de la edición de 1962, tomada de la edición inglesa de 1958. 
Otra manera de decirlo, es como lo señala directamente Popper (1980):

A partir de la obra de Hume debería haberse visto claramente que aparecen con facilidad incoherencias cuando se admite el principio de inducción; y también que difícilmente pueden evitarse (si es que es posible tal cosa): ya que, a su vez, el principio de inducción tiene que ser un enunciado universal. Así pues, si intentamos afirmar que sabemos por experiencia que es verdadero, reaparecen de nuevo justamente los mismos problemas que motivaron su introducción: para justificarlo tenemos que utilizar [nuevas] inferencias inductivas; para justificar éstas hemos de suponer un principio de inducción de orden superior y así sucesivamente. Por tanto, cae por su base el intento de fundamentar el principio de inducción en la experiencia, ya que lleva, inevitablemente a una regresión infinita (p. 29).

Así fue que Popper propuso el criterio de demarcación en ciencia, el cual establece como su norma a la falsabilidad, la cual solo es posible realizar sobre enunciados sintéticos, es decir, sobre hipótesis con pretensión científica para contrastar empíricamente. Esto es, que por medio de inferencias puramente deductivas al utilizar el Modus Tollendo Tollens, se establece una teoría $T$, de la cual se deducen consecuencias de la misma en la forma de $\mathrm{C}_{1}, \mathrm{C}_{2}, \ldots \mathrm{C}_{\mathrm{n}}$. Tales consecuencias han de ser contrastadas de forma empírica, donde la contrastación es la posibilidad de refutar $\mathrm{T}$ si los datos empíricos no coinciden con las predicciones $\mathrm{C}$, obtenidas deductivamente a partir de $\mathrm{T}$.

Como no es posible verificar de forma empírica todas las hipótesis (como sería para el caso de la inducción, pues su número es infinito), entonces lo que se hace es contrastarlas en procura de su falsación. Asunto que se resuelve con el Modus Tollendo Tollens convertido en método de investigación, descrito de forma lógica como:

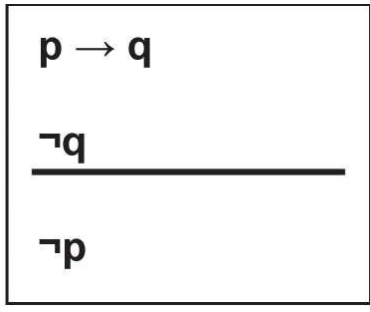


En el cual, su derivación metodológica y epistemológica como método científico de contrastación se puede ver así:

I. $\quad \mathbf{T} \longrightarrow \mathbf{h}$

Si la teoría T explica ó predice la ocurrencia de los hechos $\mathbf{h}$.

II. $\quad-\mathbf{h}$

Y si los hechos h no ocurren en las pruebas o experimentos como explica ó predice la teoría T, es decir los hechos no son ciertos.

III. $\quad-\mathbf{T}$

Por lo tanto, la Teoría T no explica ó ni predice los hechos h

Los dos primeros enunciados (I. y II.) son las premisas del razonamiento; la línea separadora señala que ha ocurrido una deducción (derivación lógica). El tercer enunciado (III.) es la conclusión, es decir, el resultado de la deducción, que es precisamente la no certeza de $\mathbf{T}$.

Esto indica la asimetría entre enunciados universales y particulares, desde el punto de vista de las propiedades lógicas del condicional presentado en I., que es la hipótesis que sustenta el criterio de universalidad, con respecto al enunciado particular que se muestra en II. Donde, tal hipótesis (enunciado universal de la forma $\mathbf{T} \longrightarrow \mathbf{h}$ ) es falsable si existe un enunciado observacional o un conjunto de enunciados observacionales lógicamente posibles (enunciados particulares -h) que sean incompatibles con dicha hipótesis, que es lo presentado en II., pues no suceden como pretende señalar el enunciado universal. Cuando esto acontece, queda falsado tal enunciado.

Una teoría o una ley científica válida es falsable justamente porque hace afirmaciones definidas acerca del mundo. Cuanto mayor sea el número de teorías conjeturadas que se enfrentan a la realidad del mundo y cuantas más abstractas sean estas conjeturas, más oportunidades habrán de hacer importantes avances en la ciencia. Esto evita la proliferación de hipótesis de especulativas, porque las teorías que sean descripciones inadecuadas del mundo y de poco potencial abarcador, pueden ser eliminadas drásticamente como resultado su refutación en la observación o en otras pruebas. 
Teniendo en cuenta el análisis del razonamiento lógico propio del Modus Tollendo Tollens, a continuación se presenta el procedimiento para contrastar teorías deductivamente. Para ello es necesario tener en cuenta los siguientes criterios:

a. Criterios lógicos: se refiere a las condiciones de coherencia y de consistencia operativa del razonamiento, en el desarrollo teórico dentro de la investigación. Las condiciones de coherencia señalan que dentro del conjunto de enunciados de la teoría, los cuales aparecen como conjunto de afirmaciones deducibles y/o deducidas, los enunciados que se derivan en la conclusión de los razonamientos, no deben ser contradictorios entre sí; y por otra parte, las conclusiones de la teoría deben ser bien deducidas. Las condiciones de consistencia indican que toda teoría científica debe tener en su estructura deductiva el rigor del procedimiento lógico, basado en el conjunto de reglas de inferencia deductiva; esto quiere decir que se supone a la teoría científica como una estructura jerárquica de enunciados, la cual tiene entre sí una organización de tal manera que de los enunciados fundamentales (los considerados evidentes por sí mismos), es decir, los axiomáticos, se derivan (deducen) enunciados en niveles de menor generalidad y categorización; y a su vez dentro de estos enunciados subordinados se dan otras deducciones entre sí. Todas estas deducciones deben tener fundamento en las reglas de inferencia deductiva.

b. Criterios teóricos: Están relacionados con la exigencia de comparar la teoría propuesta -para explicar ó predecir un conjunto de fenómenos, hechos o relación de eventos- con las otras teorías ya existentes, esto en los casos de que sea una teoría nueva. En dicha comparación es necesario ver con ojo crítico el poder explicativo ó predictivo de las distintas teorías existentes sobre el mismo tópico, de la misma manera deben verse las posibles relaciones inter-teóricas con el propósito de fusionar teorías que sean complementarias. También se refiere a la articulación de significado de los contenidos de hipótesis ad hoc (teorías adicionales a una teoría central), fusionadas con una teoría ya existente que requiere un refuerzo teórico para poder dar una mejor explicación de los hechos.

c. Criterios metodológicos de contrastación: Tienen que ver con las prescripciones procedimentales de la investigación y con las explicaciones ó predicciones sobre los resultados del estudio. En el caso de las prescripciones, se deben establecer las condiciones 
en las cuales se hace la observación y/o el experimento; que en otras palabras es la posibilidad de contrastar la teoría (mediante mediciones, observaciones, experimentos, aplicaciones de la teoría, etc.). Las explicaciones científicas de un fenómeno responden a la pregunta ¿̇cómo es que sucede éste? Y se refiere a fenómenos ya sucedidos. Las predicciones están vinculadas con la anticipación, con fenómenos que aún no han ocurrido, de acuerdo con los enunciados de la teoría, de los resultados de las observaciones. Esto se hace a través del sistema de deducciones que está relacionado con los criterios lógicos y los criterios teóricos ya mencionados. Las predicciones se manifiestan como enunciados referidos a los hechos, enunciados que son originados en la deducción a partir de la teoría que está siendo puesta a prueba. Enunciados que se llamarán aquí $\mathrm{E}_{1}$.

d. Criterios empíricos de contrastación: Estos no son cosas, ni tampoco datos. Los hechos son datos interpretados a la luz de la teoría. Estos criterios implican los diseños de la investigación, los cuales deben producir los métodos que establecen las condiciones procedimentales (metodológicas) que prescribe la teoría puesta a prueba. Las observaciones, experimentos y aplicaciones de la teoría deben darle la palabra a los hechos. Es de aquí de donde salen los enunciados referidos a los hechos. Enunciados que se llamarán aquí $\mathrm{E}_{2}$.

De acuerdo con lo anterior, contrastar empíricamente una teoría es comparar $E_{1}$ con $E_{2^{\prime}}$ esto significa que los enunciados explicativos ó predictivos derivados de la teoría $\left(\mathrm{E}_{1}\right)$ se comparan los enunciados referidos a los hechos que se dieron en la experimentación $\left(\mathrm{E}_{2}\right)$.

Puede ocurrir que $\mathrm{E}_{2}$ contradiga $\mathrm{E}_{1}$, si esto sucede entonces es necesario pensar y buscar posibles fuentes de error tales como:

- Del observador (investigador) en la observación.

- En el instrumental por ser insuficientemente preciso o sensible (validez y confiabilidad).

- En los aspectos del diseño metódico del estudio.

- En las predicciones de la teoría.

- En las prescripciones procedimentales (metodológicas) de la investigación.

- $\quad$ En los criterios lógicos de la teoría. 
Si no existen estas fuentes de error, es decir si éstas no se encuentran, entonces se puede decir que «la teoría ha sido falsada». Ello significa que la teoría que estaba siendo puesta a prueba fue refutada, pues se encontró un caso en el cual no aconteció lo que ella predecía. Aquí se aplica lo explicado en el razonamiento del Modus Tollendo Tollens; como existe un caso en el cual la teoría no funciona ni explicativa, ni descriptiva, ni predictivamente, entonces se puede afirmar que la teoría es falsa (errónea), es decir, no es sostenible para explicar ó predecir los fenómenos a los que se refiere.

También puede ocurrir que $E_{2}$ coincide con $E_{1}$, habiendo tenido en cuenta cualquiera de los errores susceptibles de cometer. En tal caso se puede afirmar que: «Todos los hechos actualmente observados $\left(E_{2}\right)$ confirman todas las explicaciones ó predicciones $\left(E_{1}\right)$ actualmente deducibles por la teoría». Es decir, la teoría ha sido corroborada empíricamente hasta el momento. Esto significa que la teoría no es falsa; pero no por ello se podrá decir que es verdadera, pues puede acontecer que aparezca un caso en el futuro que ella no pueda predecir o dar cuenta de él. Por tal razón se dice que dicha teoría es verosímil y que hasta ahora es la que mejor da cuenta de los fenómenos, en otras palabras se acepta provisionalmente.

El modelo lógico-metodológico anteriormente presentado, Popper lo tuvo en cuenta principalmente para el caso de las ciencias físico-naturales. Dicha propuesta recibió críticas y contracríticas, algunas de las cuales se expondrán más adelante.

De otra parte, hay que establecer que las ciencias, tanto las físico-naturales, como las humanas y sociales, aunque tienen un propósito común que apunta a la formulación de explicaciones por causas (eficiente o final) sobre los fenómenos que atienden; también poseen particularidades que hacen que sus diferencias en la utilización posible del método de investigación científica que se deriva del Modus Tollendo Tollens, tengan una aplicabilidad y consecuencias de distinto orden ${ }^{3}$. Para el caso de las Ciencias Humanas y Sociales y en particular las Ciencias de la Educación, se encontraran una

3 De todas formas el criterio de falsación de teorías, ha recibido muchas críticas de filósofos y científicos relacionados con las ciencias físico-naturales, pues se le considera una manera ingenua de hacer ciencia (falsacionismo ingenuo), ya que en la historia de estas ciencias no se registra un modelo de avance y progreso como el que plantea Popper. De hecho, han señalado que su monismo metodológico no se parece en nada a lo que ocurre y ha ocurrido en la historia real de las ciencias. 
serie de dificultades que se dan a conocer en el desarrollo de este trabajo, las cuales proceden de los debates sobre la historia de tales ciencias y de la crítica de las ideologías acerca de la imposibilidad de la falsación como criterio de demarcación en ciencia en general, y por ende, de ser el «método» por excelencia de las ciencias, entre ellas, las últimas citadas.

\section{Presentación del problema}

De acuerdo con lo expuesto en el apartado anterior, se puede presentar el siguiente interrogante como problema a discutir:

¿Cuál es la posibilidad lógico-metodológica que posee el Modus Tollendo Tollens que fundamenta la falsación popperiana, para realizar procesos de investigación en las Ciencias de la Educación?

\section{Presentación de la tesis}

La tesis que se sustenta para resolver el problema, plantea lo siguiente: El Modus Tollendo Tollens como regla de inferencia lógica que fundamenta el método de falsación propuesto por Popper para la contrastación de teorías en ciencia, carece de posibilidad de aplicación en la práctica de investigación en las Ciencias Humanas y Sociales y en especial en las Ciencias de la Educación: 1) porque estas ciencias son de carácter ideográfico, por lo cual no es posible establecer patrones de identidad universal entre los distintos fenómenos que ocurren y son objeto de estudio en ellas; 2) porque estas ciencias contienen dentro de los fenómenos que estudian un componente de carácter teleológico (acción humana con intencionalidad), que no permite una visión exclusivamente objetivante y controlada de los fenómenos bajo investigación, lo que impide la plena explicación por causa eficiente (erklaren), lo cual les hace requerir estudios interpretativos por causa final (verstehen) y 3) porque dentro de la historia general de las ciencias (físico naturales, lógico formales y humanas y sociales) los desarrollos de éstas nunca han evidenciado la aplicación de la propuesta falsacionista de Popper, en especial en las Ciencias Humanas y Sociales; y como consecuencia de esto, tampoco en las Ciencias de la Educación.

\section{Desarrollo de la defensa de la tesis}

A continuación se presentan tres razones por las cuales se demuestra que la falsación (uso del Modus Tollendo Tollens) como método de 
investigación en las Ciencias Humanas y Sociales y en especial, en la investigación educativa dentro de las Ciencias de la Educación, no es aplicable. Lo anterior porque:

1. Las Ciencias de la Educación como parte de las Ciencias Humanas y Sociales, son de carácter ideográfico. Esto es, que bajo un carácter descriptivo de lo individual, hacen referencia a acontecimientos o hechos particulares, que son emergentes y cambiantes, lo que impide establecer enunciados teóricos de pretensión científica universal; contraria al carácter nomotético o de leyes generales, que es propio de las ciencias físico-naturales.

Históricamente se presenta el debate planteado desde el siglo XIX al interior de la ciencia, la cual realizó una división interna de jerarquización de las disciplinas científicas, de acuerdo a sus diferentes posiciones epistemológicas. En este nuevo orden ubicó en un extremo a la matemática y a su lado, las ciencias naturales experimentales. Estas últimas, dentro de un orden descendente y determinista incluyó a la física, la química y la biología. En el otro extremo ubicó a las humanidades, con las artes y letras comenzando con la filosofía y junto a ella, el estudio de prácticas artísticas formales (literatura, pintura, escultura), dándoles un lugar cercano a la historia a través de la historia de las artes. En un punto intermedio, quedó el estudio de las realidades sociales como la historia, bajo una concepción ideográfica, cerca de las artes y las letras o como parte de ellas. Finalmente las «Ciencias Sociales», en un lugar más cerca a las ciencias naturales, bajo un carácter nomotético (Wallerstein, 2006, p. 12).

Cabe anotar que tal ubicación de las ciencias obedeció a la influencia de la filosofía positiva de Augusto Comte, la cual como tipo de conocimiento propio del último estado de la sociedad, se define por oposición a la filosofía negativa y crítica de Rousseau y Voltaire a la que Comte atribuye los males de la anarquía y la inseguridad social que caracterizan al período post-revolucionario francés de finales del siglo XVIII. Vale decir, que el término positivo hace referencia a lo real, es decir, lo fenoménico dado al sujeto. Lo real se opone a todo tipo de esencialismo desechando la búsqueda de propiedades ocultas características de los primeros estados. Lo positivo tiene como características el ser útil, cierto, preciso, constructivo y relativo (no relativista) en el sentido de no aceptar ningún absoluto. 
Como reacción Dilthey en la segunda mitad del siglo XIX rechazó abiertamente el modelo epistemológico de las ciencias de la naturaleza (Naturwissenschaften), esto es, el método científico propio de tales ciencias. Lo que le condujo a proponer el desarrollo separado de un método (hermenéutica), para las que llamó «ciencias del espíritu» (Geisteswissenschaften), es decir, aquellas áreas de conocimiento que dieran cuenta de los fenómenos relacionados con los seres humanos en su expresión individual y colectiva. Estas ciencias se fueron formando de manera individual poco a poco. Su argumento se centraba en torno a la idea de que las ciencias naturales explican los fenómenos en términos de causa y efecto (causa-efecto eficiente); por el contrario, en las ciencias del espíritu, el mecanismo fundamental para comprender los fenómenos es el principio de causa y efecto teleológico (causa final aristotélica) que conduce a estudiar los fenómenos de otra manera que permita obtener la comprensión.

Las ciencias del espíritu de Dilthey, en el siglo XX adoptaron el nombre de Ciencias Humanas y Sociales, las cuales, asumen una denominación genérica para las disciplinas o campos de saber que reclaman para sí mismas la condición de ciencias y que se ocupan de distintos aspectos de los grupos sociales y los seres humanos en sociedad, ocupándose tanto de sus manifestaciones materiales como las inmateriales. Otras denominaciones confluyentes o diferenciadas, según la intención de quien las utiliza, son las de Ciencias humanas, humanidades o letras (términos que implican distintas consideraciones epistemológicas y metodológicas).

Tales ciencias se pueden subdividir en ciencias relacionadas con la interacción social: en las cuales se encuentran: Antropología, Historia, Geografía Humana, Economía, Psicología Social, Sociología y Politología; ciencias relacionadas con el sistema cognitivo humano: Lingüística y Psicología; ciencias relacionadas con la evolución de las sociedades: Arqueología, Demografía y Ecología Humana; las humanidades: Antropología Filosófica, Ciencias de la Comunicación, Ciencia Política, Derecho, Filosofía, Periodismo, Relaciones Internacionales, Relaciones Públicas, Semiología, Sociología Jurídica, Teología y Ciencias de la Educación (que son cualquiera de las ciencias anteriormente mencionadas -que toman como objetos de investigación a fenómenos de la educación).

En el caso específico de las Ciencias de la Educación, se trata de un campo de conocimientos inherente al ser humano, el cual presenta 
diferentes disciplinas, que como ya se dijo, toman como objetos de investigación a fenómenos de la educación. Es decir, desde otras perspectivas científicas dan explicación de lo que se entiende sobre los fenómenos educativos. Tales fenómenos están involucrados en las prácticas que tienen las sociedades para reproducir sus saberes y modos de proceder, que hoy se conocen como educación. Tales fenómenos se pueden investigar desde las distintas ciencias humanas y sociales de acuerdo con sus rasgos, atributos o características a estudiar. Las cuales, están relacionadas con el carácter descriptivo de lo individual, hacen referencia a acontecimientos o hechos particulares de la educación, que son emergentes y cambiantes, lo que impide establecer enunciados teóricos de pretensión científica universal.

Lo anterior, lleva a entender que en tales ciencias se requiere utilizar métodos de investigación que sean apropiados para los fenómenos ya descritos. Lo que implica la aplicación de metodologías cualitativas de orden interpretativo y sin ninguna pretensión generalizadora. En muchos casos se utilizan métodos cualitativos de tipo inductivo, pues ante la particularidad y emergencia de los fenómenos educativos, son los procedimientos de investigación más adecuados. Esto no significa la ausencia de estudios cuantitativos, ya que estos resultan también útiles y necesarios, sino que su pretensión no será generalizadora. En esta perspectiva es totalmente inadecuado e imposible de aplicar el método de la falsación.

2. Las Ciencias de la Educación como parte de las Ciencias Humanas y Sociales, contienen un componente de carácter teleológico (acción humana con intencionalidad) que no permite una visión totalmente objetivante y controlada de los fenómenos bajo investigación, lo que impide la explicación (erklaren) plena de éstos. Lo que hace exigible la realización de procesos de investigación interpretativos, que conduzcan a la comprensión de significados e intencionalidades, aspectos vinculados con lo teleológico, es decir, entendibles por causa final (verstehen). "Droysen fue el primero que utilizó la distinción entre explicación y comprensión (en alemán Erklären y Verstehen) con intención de fundamentar el método de la historia, comprender, en contraposición al de la fisica-matemática, explicar y al de la tecnología y filosofía, conocer (erkennen). Desde entonces el término «Verstehen», comprender, viene a representar una concepción metodológica propia de las ciencias humanas» (Mardones, 1991, p. 31). 
Este debate ha estado presente desde las discusiones de planteadas por la ciencia francesa y alemana con conceptos como Naturwissenschaften frente a Geisteswissenschaften, como ya se dijo. Donde el objeto de estudio de estas ciencias incluye lo humano y su entorno, lo cual promueve que el mismo investigador, quien indaga por los fenómenos del mundo, haga parte del objeto que es investigado. Dilthey $(1949)^{4}$ señala que «El complejo de hechos espirituales que cae bajo este concepto de ciencia se suele dividir en dos miembros de los que uno lleva el nombre de «ciencias de la naturaleza»; para el otro miembro, lo que es bastante sorprendente, no existe una designación común reconocida. Me adhiero a la terminología de aquellos pensadores que denominan a esta otra mitad del globus intellectualis «ciencias del espíritu»» (p. 14).

$\mathrm{Al}$ incluir al ser humano como objeto de estudio, las Ciencias Humanas y Sociales crean la necesidad de investigar la acción humana y su intencionalidad. Desde esta perspectiva, la explicación causal apunta al pasado dentro de una relación de condición suficiente, que se contrapone a la explicación teleológica que apunta hacia el futuro, como una relación de condición necesaria. La validez de la explicación depende de la vigencia del supuesto vínculo entre causa y efecto (Von Wright, 1987, p. 107). El componente de teleológico en la acción humana con intencionalidad, limita la posibilidad de una visión totalmente objetiva y controlada de los fenómenos bajo investigación, donde la acción humana se encuentra sujeta a aspectos tanto internos como externos.

Se puede afirmar que la explicación teleológica de una conducta humana consiste en identificar en ella un objeto de intención, donde la base conceptual de la acción sea, de una parte, la ignorancia de la intervención de causas, y de otra, la convicción de que únicamente tendrá lugar determinados cambios de situación cuando se llegué a actuar. «Los acontecimientos que resultan de acciones básicas tienen lugar, por lo general, sólo cuando <investimos> estos acontecimientos de intencionalidad, i,e. ejecutamos las correspondientes acciones básicas» (Von Wright, 1987, p. 154). Lo cual, impide la explicación (erklären) plena de las causas de la acción humana sin su comprensión (verstehen).

4 Corresponde a la segunda edición en español de Introducción a las Ciencias del Espíritu de 1949. El original en alemán Einleitung in die Geisteswissenschaften data de 1883. 
Vale indicar que entre los estudios epistemológicos sobre la explicación durante el siglo XX, amerita nombrar a Meyerson, Lalande, Brunschvicg, Popper, Feigl, Oppenheim, Braithwaite, Nagel y Hempel. Sin embargo, es remarcable decir con Von Wright (1987) que «la discusión de los problemas de la explicación en el seno de la filosofía analítica [cercana y afín con el positivismo lógico] recibió un impulso decisivo del trabajo clásico de Carl Gustav Hempel, The Function of General Laws in History, publicado por el Journal of Philosophy en 1942» (p. 29).

Prosigue esta línea del argumento diciendo que cuando se hace referencia a explicación (erklären) es la condición de causalidad que busca la conexión objetiva entre antecedente-consecuente, a fin de seguir la evolución del proceso sobre el cual se desenvuelve el fenómeno a analizar. La explicación (erklären) se expresa dentro de un sustento teórico en forma de hipótesis coherente dentro del conocimiento científico, a fin de evitar especulaciones o meras descripciones, para permitir la construcción de teorías validadas de manera empírica. En términos de Von Wright (1987) la explicación por cobertura legal se puede definir así:

Sea $E$ un acontecimiento cuya ocurrencia en alguna ocasión es conocida y se ve precisada de explicación. ¿̇Por qué ha ocurrido $E$ ? Con vistas a responder esta cuestión, indicamos algún otro acontecimiento o estado de cosas determinado $E_{1}, \ldots, E_{m^{\prime}}$ y apuntamos una o varias proposiciones generales o leyes $L_{1}, \ldots, L_{n}$, tales que la ocurrencia de $E$ (en el momento considerado) se sigue lógicamente de esas leyes y del hecho que de aquellos otros acontecimientos (estados de cosas) haya ocurrido (se hayan dado) (p. 31).

En este contexto, la causalidad responde a correlaciones de fenómenos, los cuales son posibles de registrar a partir de escalas. Dicha explicación guarda un orden epistemológico correspondiente a la dinámica entre pensamiento-lenguaje-interacción que se concreta en el establecimiento de teoría y metodología. Es por así decirlo, des-enrollar de manera ordenada y sistemática una serie de explicaciones causales, en la forma de correlación estadística, a fin de justificar desde lo empírico una ley o teoría que pretende dar razón de un fenómeno dado. Esto supone una hipótesis ontológica subyacente, consistente en considerar la uniformidad de los fenómenos de la naturaleza y de la sociedad. 
La comprensión (verstehen) parte de tal estado de cosas en el que todo desempeño individual no solo es la expresión de una dinámica interna de la conciencia de los sujetos, sino que además posee sentido (significación). Tal comprensión procura interpretar la orientación de la acción. (Ursúa Lezaún et.al., 1981, p. 151). De esto se deriva el conocimiento construido a través de un proceso de interpretación de los fenómenos propios de la vida humana registrados en la conciencia, el cual se caracteriza porque devela el significado y la intencionalidad de ésta, dentro de un contexto histórico-social-cultural. Es la interpretación (hermenéutica) por lo tanto, el método más adecuado para producir los conocimientos sobre los fenómenos que tratan las Ciencias Humanas y Sociales. Así que los fenómenos que se evidencian «en la realidad», requieren de un proceso de comprensión en el orden científico cultural, donde los comportamientos individuales de los fenómenos no son solo la exteriorización de un proceso interno, sino que además tiene un significado factible de analizar a partir de la regularidad con que se presentan en la acción.

La comprensión como forma de aprehensión o expresiones del espíritu es el acto por el cual se conoce en su interioridad la conciencia a través de sus múltiples exteriorizaciones. Además,

Comprender e interpretar textos no es sólo una instancia científica, sino que pertenece con toda evidencia a la experiencia humana del mundo. En su origen el problema hermenéutico no es en modo alguno un problema metódico. No se interesa por un método de la comprensión que permita someter los textos, igual que cualquier otro objeto de la experiencia, al conocimiento científico. Ni siquiera se ocupa básicamente de constituir un conocimiento seguro y acorde con el ideal metódico de la ciencia. Y sin embargo trata de ciencia, y trata también de verdad (Gadamer, 1988, p. 23).

Teniendo en cuenta lo argumentado en el presente literal, se infiere que no es posible utilizar el Modus Tollendo Tollens como método de falsación de teorías en las Ciencias Humanas y Sociales y en las Ciencias de la Educación, ya que la deducción lógica que se observa en la negación del consecuente (el fenómeno que es explicado por una teoría) de la primera premisa del Modus Tollendo Tollens, representado en la segunda premisa, para concluir en la negación del antecedente, es decir, la teoría misma, es imposible en 
concepciones epistemológicas que consideran la intención de los individuos dentro de los fenómenos estudiados, como sucede en las epistemologías comprensivas de las ciencias ya señaladas. Lo cual, no permite una explicación por causa eficiente (erklaren), donde hay, sí o no, un antecedente que deriva un consecuente de manera empírica y probable, generalmente en términos estadísticos. Sino que hay unas interacciones que generan un escenario, en el cual, lo que acontece como causa, tiene internamente unos significados e intereses o intenciones de protagonistas que sobrepasan las posibilidades metódicas de la falsación; y es necesario recurrir a la interpretación (círculo de la interpretación para la comprensión) para que a través de la empatía con los textos o los plexos de sentido (escenarios, circunstancias, íconos, obras, etc.) que son huellas o registros de lo que los seres humanos han plasmado, poder develar dichos significados y las pretensiones últimas (causa final) inmersas, que generan determinadas consecuencias relacionadas con la acción en el mundo objetivo, social o subjetivo, cuando se aborda un fenómeno educativo, como objeto de investigación.

3. Se observa dentro de la historia general de las ciencias (físiconaturales, lógico-formales y humanas y sociales) que los desarrollos de éstas, nunca han evidenciado la aplicación de la propuesta epistemológica-metodológica de Popper. Es decir, que en el desarrollo de las teorías científicas en las distintas ciencias, en especial en las Ciencias Humanas y Sociales y como consecuencia de esto, tampoco en las Ciencias de la Educación, ha operado el método de falsación de teorías. Lo cual significa que el monismo metodológico de la aplicación del Modus Tollendo Tollens, para explicar el avance de las ciencias nunca ha ocurrido. Lo cual niega de manera empírica e histórica la validez de tal método para hacer ciencia real.

La propuesta popperiana, se enfrenta a la crítica desde dos perspectivas: Una de orden lógico y otra de orden metodológico (que necesariamente implica lo epistemológico). Respecto al carácter lógico, se evidencia que tal método racional hipotético-deductivo no da cuenta del desarrollo del conocimiento científico; y desde el carácter metodológico, las prácticas científicas que se llevan a cabo en el contexto de lo real, no se ajustan al método de la falsación. Otro aspecto de orden lógico, es la relación entre enunciados en una ley científica, los cuales son posibles de falsear, más no de verificar; mientras que desde lo metodológico, siempre es posible poner en duda un enunciado de observación. 
Habrá que tenerse en cuenta, que en la observación se puede presentar algún error en la observación registrada o puede que el objeto, como también es factible que no haya sido correctamente delimitado. Así que, siempre es posible rechazar, sin entrar en contradicción, la validez de un enunciado de observación, tal como se explicó en la Introducción. Estos argumentos, llevan que se descarte cualquier experiencia falsadora. Como también, se pueden reinterpretar los hechos observados para adecuarlos a los enunciados, cuando en la contrastación falsadora de alguna manera se amolden los enunciados sobre los hechos a los enunciados de la teoría bajo prueba, situación que conduce a un enfoque acientífico, ya que éste no es el proceder adecuado en la práctica ética de la ciencia.

En el ambito metodológico, la constratación de la hipótesis dentro de la falsación considera a la observación como fiable y unívoca. Sustenta la prueba del proceso de falsación en observaciones consideradas como fiables, sin tener en cuenta que la percepción de lo real, está influenciada por factores subjetivos, ambientales o culturales, así se tengan de presente los críterios lógicos, teóricos, metodológicos y empíricos presentados al inicio de este ensayo. Aquí se suma el hecho, de que la falsación obedece a situaciones complejas, ya que una teoría está constituida por un conjunto de enunciados, axiomas o prinicipos, los cuales no necesariamente quedan cuestionados en la falsación de una hipótesis. Así que, el falsacionismo, basado en la observación y la experimentación, no puede contrastar negativamente ninguna teoría, ya que el conocimiento científico no se desarrolla en forma lineal o frontal (Rodas Malca, 2004, p. 95).

Se reconoce sin embargo, que la intención de Popper con su propuesta falsacionista, basada en el Modus Tollendo Tollens, es generar una actitud crítica frente a las teorías científicas, ya que es gracias a la crítica, como ha sido posible avanzar la ciencia, pero, que se requiere de una normatividad desarrollada por los mismos científicos, que no existe de manera clara y hace difícil definir los límites de las observaciones y su conversión a enunciados sobre los hechos; y de ahí ponerlos en contraste con los enunciados de teoría eludiendo intereses personales, económicos e institucionales. A pesar de ello, el falsacionismo, siendo crítico, no puede explicar el avance de la ciencia, porque ésta no ha progresado por falsaciones. 
Ahora, desde la controversia entre Kuhn y Popper en el Coloquio Internacional de Filosofía de la Ciencia de 1965 en Londres, se encuentra la crítica que presentó Thomas Kuhn (1922-1996), basado en su texto de La Estructura de las Revoluciones Científicas (1962)5. En primer lugar, le da un papel de importancia a la historia como telón de fondo en el cual se desenvuelve la actividad científica. «Si se considera a la historia como algo más que un depósito de anécdotas o cronología, puede producir una transformación decisiva de la imagen que tenemos actualmente de la ciencia». (p. 20). En dicha obra presenta una distinción entre ciencia normal y ciencia extraordinaria, como parte de los desarrollos a través de la historia de las ciencias, en los cuales introduce el concepto de paradigma, inicialmente entendido, con imprecisión, como elaboraciones científicas universalmente reconocidas, las cuales, durante un período de tiempo proporcionan modelos de problemas y soluciones a las comunidades científicas. Algunos de sus críticos llegaron a decir que utilizó el término dentro de la citada obra con 21 significados diferentes.

Kuhn respondió en una publicación de $1969^{6}$ donde restringe tamaña cantidad de alternativas a sólo dos: en la primera el paradigma es sinónimo de "Ejemplar» - una teoría modelo aceptada por una comunidad científica como guía de su investigación durante un período de tiempo, por ejemplo la dinámica newtoniana. La segunda acepción es más global y Kuhn la denominó «Matriz Disciplinaria», la cual significa la integración en un solo cuerpo teórico, de los diversos elementos, que por separado se encuentran dispersos con escasa relación funcional y poder explicativo limitado, que se mantienen dentro de una comunidad de científicos que profesan una disciplina de investigación en un campo de conocimientos científicos específico. Tal delimitación, se logra a través de la inspección de los paradigmas que la conforman. Así que la ciencia normal se encarga de la resolución de enigmas instrumentales, conceptuales y matemáticos. Contexto en el cual se forma el experto, quien después de prepararse en el estudio de los paradigmas o matrices disciplinares compartidos por la comunidad científica, logra con éxito resolver los enigmas planteados. Los científicos de la ciencia normal, se apoyan en el paradigma, buscan determinar los hechos

5 Aquí se utiliza la octava reimpresión (2004) de la primera edición en español de 1971.

6 Kuhn, Thomas. Posdata: 1969. En: La estructura de las revoluciones científicas. FCE. México. 1971. pp. 287-293. 
significativos, acoplan los hechos con la teoría y articulan la teoría para su posterior publicación (Jaramillo y Aguirre, 2004, sp.). De tal manera que el científico normal intenta adecuar la naturaleza dentro de los moldes impuestos por el paradigma. Aspecto que confirma que la ciencia normal no tiende hacia novedades fácticas.

En el ámbito de la ciencia, el rechazar un paradigma, conlleva a que otro lo remplace, hasta el grado en que se dedique a la ciencia normal. Esto demuestra que el investigador es un solucionador de enigmas, no alguien que pone a prueba los paradigmas; para Kuhn, los fracasos no rechazan del todo las teorías, es suficiente con introducir hipótesis ad hoc. (Jaramillo y Aguirre, 2004, sp.). Afirma Kuhn (2004), que existe una serie de fenómenos que no se dejan asimilar por los paradigmas existentes, pues en ocasiones no son percibidos por los científicos de la ciencia normal; a estos fenómenos los llama anomalías, consistentes en un no acoplamiento con el paradigma existente y se presentan como un enigma más de la ciencia normal. «... la investigación científica descubre repetidamente fenómenos nuevos e inesperados y los científicos han inventado, de manera continua, teorías radicalmente nuevas» (Kuhn, 2004, p. 92).

Esta dificultad, hace que un mayor número de científicos intenten resolverlo a través de hipótesis ad hoc, lo que genera confusiones y discusiones entre los científicos de la ciencia normal. Cuando un paradigma o matriz disciplinar está lleno de hipótesis ad hoc, significa que está en crisis, lo que conduce a los mas jóvenes miembros de la comunidad científica a buscar nuevas respuestas teóricas unificadas, que superen la teoría actual llena de anomalías cubiertas por hipótesis ad hoc. Esto conduce a la proliferación de respuestas nuevas que son potencialmente sustitutas de la teoría vigente, y entre ellas, se adopta la que presente mayor alcance explicativo que lleve al derrumbe del paradigma vigente y genere un cambio por uno nuevo que se construya alrededor de la nueva teoría.

De acuerdo con lo anterior, Jaramillo y Aguirre (2004, sp.) haciendo referencia al debate entre Kuhn y Popper en el Coloquio Internacional sobre Filosofía de la Ciencia, realizado en Londres en 1965, señalan que Popper solo toma una sola cara de la moneda, al tener como argumento el «derrocamiento revolucionario» de una teoría aceptada, mediante procesos de falsación de la teoría vigente (asunto que de manera clara no ha sido registrado en la historia de 
la ciencia), que luego ha sido reemplazada por otra mejor; situación que hace que desconozca la distinción entre ciencia normal y ciencia extraordinaria, esta última referida al cambio por la nueva teoría vigente. Según Kuhn, Popper sólo analiza lo que ocurre en la ciencia en tiempos de crisis, pero olvida la práctica «normal» de la ciencia. Argumentos que Popper controvierte, diciéndo que no puede ser tajante la distinción entre ciencia normal y ciencia extraordianria. Además, asegura que ninguno de los científicos registrados en los anales de la historia de la ciencia, fueron «científicos normales». En esta polémica terció Imre Lakatos con su teoría de la Metodología de los Programas de Investigación en la que media entre el falsacionismo ingenuo de Popper y el historicismo de $\mathrm{Kuhn}^{7}$.

En relación a las críticas de Feyerabend (1924-1994) hacia el falsacionismo se puede decir que:

La evolución de su pensamiento tuvo lugar al hilo de sus trabajos sobre la teoría de la medida y la lógica cuántica. Se fue interesando crecientemente por las reglas metodológicas que los científicos tienen que usar para sus pruebas. Feyerabend observó que aquellas eran violadas por los propios científicos, en oposición a las tesis empiristas. Asimismo, si hay que atenerse al criterio popperiano de falsabilidad, las teorías alternativas a una teoría que haya sido verificada muchas veces, pueden producir hechos capaces de falsar esta última y, por tanto, son indispensables para su examen. Una vez en este camino se va desvaneciendo la línea de demarcación entre ciencia y no ciencia. La insistencia en la demarcación disminuye el contenido empírico de la ciencia y lo hace más dogmático (Hernández et.al., 2005, sp.).

Estas críticas fueron muy duras, pues Feyerabend (1986), quien trabajó con Popper, con su estilo mordaz consideró dogmático y arrogante al falsacionismo. «La historia en general, y la historia de las revoluciones en particular, es siempre más rica en contenido, más variada, más multilateral y más viva e ingeniosa de lo que incluso el mejor historiador y el mejor metodólogo [posiblemente se refiere a Popper] pueden imaginar»(p. 1). Su defensa del todo vale surge justamente porque los científicos a lo largo de la

$7 \quad$ Las memorias de este coloquio, según Jaramillo y Aguirre (2004, sp.) se deben al esfuerzo de Lakatos y Musgrave, quienes las editaron en 1970.

ITINERARIO EDUCATIVO • ISSN OIZI-2753 • AÑO XXVII, N. ${ }^{\circ}$ GI • ENERO - JUNIO DE ZOI3 • P. I8I-ZO8 
historia no han mantenido una disciplina de racionalidad en las metodologías aplicadas. Se pregunta si vale creer si las ingenuas reglas que los metodólogos tienen como guía (parece volver aquí contra Popper) son suficientes para explicar el laberinto de las interacciones del mundo (Feyerabend,1986, p.2). Enfatizó en que «la idea de un método que contenga principios firmes, inalterables y absolutamente obligatorios que rijan el quehacer científico tropieza con dificultades considerables al ser confrontada con los resultados de la investigación histórica. Descubrimos entonces, que no hay una sola regla, por plausible que sea, y por firmemente basada que esté en la epistemología, que no sea infringida en una ocasión u otra» (Feyerabend, 1986, p. 7). Por eso su propuesta del todo vale.

Coincide con lo anterior, que la aplicación de la propuesta de Popper, no se ha evidenciado, en el caso de las Ciencias Humanas y Sociales y como consecuencia de esto, tampoco en las Ciencias de la Educación. Esto se puede ver en el debate entre Adorno y Popper, respecto a la disputa del positivismo en la sociología alemana. Donde es contundente la postura de Adorno, a través de la «Teoría Crítica de la Sociedad», la cual, según Adorno, el espíritu crítico en general y el particular dicho espíritu para la práctica científica, especialmente la de las Ciencias Sociales, se efectúa en la auto-comprensión en el desarrollo de las contradicciónes de la sociedad mediante el conocimiento de las mismas. En Popper, por el contrario, la categoría de la crítica está reducida a prescripciones lógicas y metodológicas. Tan solo se refiere a mecanismos para la confirmación provisional de enunciados generales de la ciencia (Dahrendorf, 1973, p. 140).

Relacionado con lo anterior y con respecto a la concepción y uso de los métodos, respecto de los fenómenos objeto de investigación, Dahrendorf (1973), citando a Adorno, señala:

...extraigo una consecuencia de la que no se habla en la ponencia de Popper y que ignoro si estaría dispuesto a asumir. Da a su punto de vista el calificativo de criticista, en un sentido nada kantiano. Si se interpreta la dependencia del método [falsación] respecto de la cosa con el mismo rigor con el que viene implícita en algunas determinaciones popperianas, como la de la relevancia y el interés como patrones de medida del conocimiento social, no le sería posible al trabajo crítico de la sociología limitarse a la autocrítica, a la reflexión sobre sus enunciados, 
teoremas, métodos y aparatos conceptuales. Es al mismo tiempo crítica del objeto [de investigación] del que dependen todos estos momentos, localizados en el lado subjetivo, en el de los sujetos vinculados a la ciencia organizada. Por muy instrumentalmente que sean definidos los momentos metodológicos, su adecuación al objeto viene exigida siempre, aun cuando a veces sólo de manera velada. Los métodos sólo son improductivos cuando les falta esta adecuación. La cosa debe gravitar con todo su peso en el método, y ostentar en él su propia vigencia; de lo contrario, incluso el método más depurado resulta deficiente (p. 130).

Así que, dentro de la discusión que sostiene Popper y Adorno, respecto a la relación entre teoría y experiencia para las ciencias, en particular para las Ciencias Humanas y Sociales, Popper lo sustenta en el método hipotético-deductivo (falsación), bajo el supuesto de que no hay observación sin hipotesis. En cambio para Adorno, la relación entre teoría e investigación empírica es algo más complejo, por la divergencia entre el concepto de una teoría crítica de la sociedad y la investigación social empírica, que lleva a la aplicación del primero sobre el segundo. Así que la teoría crítica de la sociedad, le plantea a la investigación empírica, una enorme cantidad de cuestiones, las cuales por sí misma no es capaz de cristalizar (Dahrendorf, 1973, p. 142). Esta controversia que es epistemológica, lleva implícita la crítica metodológica y lógica a la aplicación del Modus Tollendo Tollens.

En consecuencia, las Ciencias Humanas y Sociales y en particular en las Ciencias de la Educación, no existen ejemplos detallados sobre cómo aplicar el método hipotético-deductivo de la falsación. El caso más desarrollado, se encuentra en el texto sobre la teoría del progreso científico e industrial, al que Popper dedica el capítulo 32 de la obra La Miseria del Historicismo (Molini Fernández, s.f., p. 3). Donde la aplicación de su método, se limita a hacer un análisis teórico de «las condiciones bajos las cuales el progreso se detendría». Aquí aboga por el progreso científico como resultado de la libre competencia del pensamiento, pero queda por fuera la evaluación de su método de falsación. Concluye que el progreso depende, en gran medida, de factores políticos, de insituciones políticas, lo cual autorefuta su tesis falsacionista sobre el progreso de la ciencia. Argumento con el cual, se evidencia que todo lo expuesto en su teoría es muy 
reducido, al reconocer componentes más complejos del mundo de la vida científica; tan solo se queda con una intención de «monismo metodológico», en la que paradójicamente cae Popper, y que no intenta falsarla (Molini Fernández, s.f., p. 3).

Así también, las evidencias en la historia, de que la ciencia avanza gracias a las condiciones políticas y sociales, más allá de las prescripciones metodológicas, queda reafirmada por los acontecimientos evidenciados en la decada de los años 50 del siglo XX, donde se dieron grandes avances científicos, aún sin estar presentes la libertad de pensamiento y la democracia, dentro de sistemas políticos autoritarios en los países socialistas dominados por la URSS, así como en los países de las democracias representativas en las naciones líderes del capitalismo occidental.

En relación con los argumentos de Jürgen Habermas sobre la teoría y la práctica en las ciencias sociales, no es posible formular predicciones sobre el futuro histórico. Aspecto que aplica para la metodología de la investigación científica dentro de las Ciencias Humanas y Sociales y en particular para las Ciencias de la Educación, ya que tan solo se pueden realizar recomendaciones para la acción, la cual «se impone gracias a la voluntad y a la consciencia de los hombres y no de manera «objetiva», con lo que no pueden ser anticipadas, calculadas y previstas, sino sólo en condiciones objetivas de posibilidad (Habermas, Teoría y Praxis, p. 303 citado por Pilot, 1973, p. 288). «El «sentido de la historia» no es, pues, otra cosa que el posible futuro de la misma, realizable mediante la acción, «La filosofía experimental de la historia ya no busca un Sentido oculto; lo salva en la medida en que lo produce»» (Pilot, 1973, p. 288).

Así que, dentro de la lógica de las Ciencias Humanas y Sociales y en particular para las Ciencias de la Educación, tan solo se presentan argumentos de orden «teorético», basados en un aparato crítico de la sociedad, con lo cual, hacen de su objeto de investigación, un aparato categorial de interpretación en sí mismo. Desde el punto de vista metodológico, el camino que recorren cada una de las ciencias son inconmensurables, razón por la cual, sus historias son complementarias, habiendo algunos puntos en común sobre los métodos usados en la investigación de las mismas, pero también la delimitación de los alcances y deficiencias de los métodos de las ciencias de la naturaleza cuando se han aplicado a las ciencias Humanas y Sociales. 
Según Wallerstein (2006), la mayoría de los investigadores y de la indagación académica en el período entre 1850 y 1914, factiblemente hasta 1945, proviene tan sólo de cinco países: Francia, Gran Bretaña, las Alemanias, las Italias y los Estados Unidos. Habrá que considerar aquí el papel colonialista de la mayoría de estos países y la emergencia como potencia de Norteamérica. Es allí donde a lo largo de la segunda década del siglo XIX se van estableciendo en las universidades unidades administrativas para organizar los estudios sobre los distintos fenómenos humanos y de la sociedad en general. Esto por la disciplinarización y profesionalización del conocimiento que llevó a establecer estructuras institucionales permanentes para producir nuevo conocimiento y reproducir productores de conocimiento. Hay algo más; no sólo la investigación académica se hacía en estos cinco países, sino que gran parte de la investigación era hecha por la mayoría de los estudiosos sobre su propio país y se abría para el caso de las colonias. Esto fue parcialmente pragmático y en parte obedeció a presión social y a razones ideológicas, políticas y culturales: eran los países importantes, lo que interesaba y lo que se debía estudiar era para saber cómo operaba el mundo, para continuar la dominación o para ampliar nuevos frentes de conquista. El nombre dado a las disciplinas científicas sociales y humanas, fue más por motivos económico-políticos-administrativos que por motivos epistemológicos o metodológicos. Esto debido a la formación de facultades, departamentos y unidades de investigación en las universidades.

Los avances o las formulaciones de teorías obedecieron a propuestas especulativas que eran debatidas desde lo filosófico y desde la experiencia. Pero nunca desde la aplicación de métodos asociados a la falsación. Imperaba más el racionalismo, el empirismo y el idealismo atravesados por el debate crítico de la filosofía kantiana, como fundamentos gnoseológicos de estas nacientes ciencias, que originalmente se llamaron humanidades o ciencias morales, en inglés y en las lenguas romance. En la cultura alemana, como ya se dijo, fue diferente, pues Dilthey propuso el nombre de ciencias del espíritu para los estudios concernientes a lo humano-social, de donde surge la hermenéutica como epistemología y metodología de investigación para tales ciencias. La denominación de Ciencias Sociales o Ciencias Humanas y Sociales se establece en el siglo XX luego de 1945. Con la descripción de este desarrollo histórico se demuestra que en estas ciencias, su recorrido, nada tiene ver con el Modus Tollendo Tollens como regla lógica que inspira al método de la falsación propuesto por Popper. 


\section{Conclusiones}

Teniendo en cuenta lo expuesto, se reafirma que lo planteado en la tesis, respecto a que el Modus Tollendo Tollens como regla de inferencia lógica que fundamenta el método de falsación propuesto por Popper para la contrastación de teorías en ciencia, carece de posibilidad de aplicación en la práctica de investigación en las Ciencias Humanas y Sociales y en especial en las Ciencias de la Educación.

Lo anterior, se fundamenta en: (1) El reconocimiento del carácter descriptivo de lo individual, referente a acontecimientos o hechos particulares, que son emergentes y cambiantes, lo que impide establecer enunciados teóricos de pretensión científica universal que pudieran ser contrastados por falsación en tales ciencias. (2) Existen unas interacciones que generan un escenario, en el cual, lo que acontece como causa, tiene internamente unas intenciones y significados de protagonistas que sobrepasan las posibilidades metódicas de la falsación; y es necesario recurrir a la interpretación para que a través de la empatía con los textos que reflejan los sujetos de las relaciones, poder develar las pretensiones últimas (causa final o telos) de los actores que generan determinadas consecuencias en el mundo de lo social o educativo, como objeto de investigación, y (3) La lógica de las Ciencias Humanas y Sociales y en particular para las Ciencias de la Educación, tan solo se presentan argumentos de orden «teorético», basados en un «aparato crítico de la sociedad» el cual se constituye en objeto de investigación en sí mismo, que obliga pasar de la comprensión crítica a la crítica comprensiva para la transformación de la sociedad. Y la historia de la configuración de las Ciencias Humanas y Sociales desde el siglo XIX ha estado plagada de desarrollos vinculados con intereses colonialistas y administrativos, más que epistemológicos; motivos por los cuales, no existe una evidencia en la historia de dichas ciencias que demuestre la aplicación del Modus Tollendo Tollens, como posibilidad metodológica para realizar llevar a cabo investigaciones de orden empírico-falsable.

\section{Referencias}

Dahrendorf, R. (1973). Anotaciones a la discusión de las ponencias de Karl R. Popper y Theodor W. Adorno. En: La disputa del positivismo en la sociología alemana (pp. 139-146). Ediciones Grijalbo. México D. F.: Barcelona. 
Dilthey, W. (1949). Introducción a las ciencias del espíritu. México: Fondo de Cultura Económica.

Feyerabend, P. (1986). Tratado contra el Método. México: Editorial Tecnos.

Gadamer, H. G. (1977). Verdad y Método. Salamanca: Ediciones Sígueme.

Hernández Fernández, L. R., et. al. (2005). Tesis Básicas del Racionalismo Crítico. En revista Cinta de Moebio. No. 23. Universidad de Chile. Santiago. sp.

Jaramillo, L. G. y Aguirre, J. C. (2004). La Controversia Kuhn-Popper en torno al progreso científico y sus posibles aportes a la enseñanza de las ciencias. En revista: Cinta de Moebio, No. 20, Facultad de Ciencias Sociales, Universidad de Chile. Santiago. s.p.

Kuhn, T. (2004). Estructura de las Revoluciones Científicas. México: Fondo de Cultura Económica.

Mardones, J. M. (1991). Filosofía de las ciencias humanas y sociales. Materiales para una fundamentación científica. Barcelona: Anthropos Editorial.

Molini Fernández, F. (1999). Proactividad: El método científico de Karl Popper aplicado al futuro. Encuentros multidisciplinares. Vol. 1 No. 3. Universidad Autónoma de Madrid, pp.1-12.

Ochoa Rojas, L. F. (2013). Hacia una teoría de relaciones epistemológicas en investigación educativa. Bogotá.

Pilot, H. (1973). La filosofía de la historia empíricamente falsable de Jürgen Habermas. En La disputa del positivismo en la sociología alemana (pp. 287-312). Barcelona - México D. F.: Ediciones Grijalbo.

Popper, K. (1980). La Lógica de las Investigación Científica. Madrid: Tecnos.

Rodas Malca, A. (2004). ¿Cómo desarrollan las ciencias? En: Revista de Educación, Cultura y Sociedad, FACHSE (UNPGR) Lambayeque, Año IV, No. 7, pp. 94-100.

Ursúa Lezaún, N., et. al. (1981) Filosofía de la Ciencia y Metodología Crítica. Bilbao: Desclée de Brouwer.

Von Wright, G. H. (1987). Explicación y Comprensión. Madrid: Alianza Editorial. 
Wallerstein, I. (2006). Abrir las ciencias sociales. Informe de la comisión Gulbenkian para la reestructuración de las ciencias sociales. México: Siglo XXI. 